\title{
Gait Patterns and Mood in Everyday Life: A Comparison Between Depressed Patients and Non-depressed Controls
}

\author{
Dirk Adolph $^{1}$ (D) $\cdot$ Wolfgang Tschacher ${ }^{2} \cdot$ Helen Niemeyer $^{3} \cdot$ Johannes Michalak $^{4}$
}

Accepted: 8 February 2021 / Published online: 25 February 2021

(c) The Author(s) 2021

\begin{abstract}
Background Previous laboratory findings suggest deviant gait characteristics in depressed individuals (i.e., reduced walking speed and vertical up-and-down movements, larger lateral swaying movements, slumped posture). However, since most studies to date assessed gait in the laboratory, it is largely an open question whether this association also holds in more naturalistic, everyday life settings. Thus, within the current study we (1) aimed at replicating these results in an everyday life and (2) investigated whether gait characteristics could predict change in current mood.

Methods We recruited a sample of patients $(n=35)$ suffering from major depressive disorder and a sample of age and gender matched non-depressed controls $(\mathrm{n}=36)$. During a 2-day assessment we continuously recorded gait patterns, general movement intensity and repetitively assessed the participant's current mood.

Results We replicated previous laboratory results and found that patients as compared to non-depressed controls showed reduced walking speed and reduced vertical up-and-down movements, as well as a slumped posture during everyday life episodes of walking. Moreover, independent of clinical diagnoses, higher walking speed, and more vertical up-and-down movements significantly predicted more subsequent positive mood, while changes in mood did not predict subsequent changes in gait patterns.

Conclusion In sum, our results support expectations that embodiment (i.e., the relationship between bodily expression of emotion and emotion processing itself) in depression is also observable in naturalistic settings, and that depression is bodily manifested in the way people walk. The data further suggest that motor displays affect mood in everyday life.
\end{abstract}

Keywords Embodiment $\cdot$ Depression $\cdot$ Gait patterns $\cdot$ Ambulatory monitoring $\cdot$ Everyday life

\section{Introduction}

In basic research, the interaction between the motoric system and emotional processes has been extensively explored during the past two decades. For example, a large number of studies have investigated the effects of emotional processes

Dirk Adolph

dirk.adolph@rub.de

1 Mental Health Research and Treatment Center, RuhrUniversity Bochum, Massenbergstraße 9-13, 44787 Bochum, Germany

2 University Hospital of Psychiatry and Psychotherapy, University of Bern, Bern, Switzerland

3 Department of Education and Psychology, Freie Unversität Berlin, Berlin, Germany

4 Department of Psychology and Psychotherapy, Witten/Herdecke University, Witten, Germany on the motoric system (e.g., posture, facial expression) as well as the reverse effects of the motoric system on emotional processes (Winkielman et al. 2015). Contradicting recent doubts regarding the robustness of a number of these effects typically referred to as 'embodiment' (Simmons and Simonsohn 2017; Wagenmakers et al. 2016), meta-analyses have shown that motor displays affect a broad range of emotional processes (Cuddy et al. 2018; Elkjær et al. 2020). Embodiment theories of emotion postulate that emotional states (i.e., current mood) are consistently accompanied by multimodal reproduction or simulation of experiential and motor states (Winkielman et al. 2015). For example, while getting sad, traces of direct perceptual experiences including motor states associated with sadness are re-activated. Moreover, specific motor states can further activate associated information in the multimodal emotion network. Therefore, the hereby established bodily and cognitive feedback loops stabilize the current emotional state (Teasdale and Barnard 
1993). However, until now, most embodiment studies have investigated effects in highly controlled laboratory settings, leaving open the question whether motoric processes are also relevant for emotions in everyday life. Within the current study, we aimed at closing this gap and investigated the association of embodiment processes and current mood in everyday life in a sample of non-depressed controls and a matched sample of patients suffering from major depressive disorder (MDD).

Indeed, most studies thus far investigated embodiment in non-clinical populations, and to the best of our knowledge, no study to date has investigated individual differences in the association of embodiment processes (specifically gait characteristics) and emotional states in everyday life between non-depressed individuals and people suffering from MDD. Several lines of research suggest embodiment processes to be highly relevant for understanding cognitive and emotional processes in MDD. Only recently, more attention has been drawn on research on embodiment effects in the context of psychological disorders, mostly with respect to major depressive disorder (MDD), but also other disorders such as schizophrenia (Tschacher et al. 2017; Lyons et al. 2021). Some of these studies have focused on the effects of the motoric system on memory bias in major depressive disorder (MDD). A bias towards the recall of negative, relative to positive, information in MDD is one of the most robust findings so far (Mathews and MacLeod 2005; Matt et al. 1992). For example Michalak et al. (2014) found that patients suffering from MDD showed the typical negative memory bias when sitting in a slumped posture. However, when patients sat in a 'non-depressed' upright posture their recall of positive and negative words was unbiased. In another study with MDD patients an upward-opening Qi Gong movement, which runs counter to the habitual slumped and downward depressive movement style, or a downwardclosing Qi Gong movement was used (Michalak et al. 2018). Qi Gong is an ancient Chinese system using coordinated movements to increase physical and mental well-being. Patients in the upward-opening movement condition, in contrast to the downward-closing movement condition, showed a more positively biased recall of affective words. In addition, a number of studies have investigated gait patterns of patients with MDD in the laboratory (Lemke et al. 2000; Paleacu et al. 2007; Sloman et al. 1982; 1987), supporting differences in gait patterns between non-depressed controls and depressed patients. A comprehensive laboratory-based 3-dimensional analysis of gait demonstrated that patients suffering from MDD in contrast to never-depressed control participants displayed reduced walking speed, smaller arm-swing amplitudes, smaller amplitude of vertical movements of the upper body, ${ }^{1}$ larger amplitudes of lateral body sway, and a more slumped and forward-leaning posture than non-depressed controls (Michalak et al. 2009). An animation of depressed gait patterns can be found at http://biomo tionlab.ca/Demos/BMLdepression.html). Using this data, Michalak et al. (2015), gave (non-clinical) participants biofeedback that modified their gait patterns. This gait modification was associated with an affective memory bias. That is, participants who walked 'happier' than usual showed a pronounced tendency to recall more positive than negative words, whereas the difference between recalled positive and recalled negative words was much lower in participants who adopted a depressed walking style. Although the findings presented above suggest that the motoric system might be relevant for the maintenance of MDD, they are based exclusively on laboratory research and only covered relatively short periods of walking.

In sum, the goal of our present research was twofold. First, we aimed to replicate the laboratory findings on gait characteristics of depressed individuals (Michalak et al. 2009). We used an ambulatory device to record gait parameters over two days expecting that depressed individuals, compared to never-depressed individuals, should show reduced walking speed, reduced vertical up-and-down movements of the upper body, larger lateral swaying movements of the upper body and a slumped posture. Second, to obtain a better understanding of the dynamics of body-emotion interaction, we investigated whether gait characteristics could predict mood during the 2-day period and whether the strength of this association might differ between patients suffering from MDD and non-depressed controls. Specifically, previous research has found a stronger association between physical activity and subsequent mood in MDD patients as compared to non-depressed controls (Mata et al. 2011). Within the present study, we aimed at investigating whether this stronger relationship would also hold for the association between gait and mood.

\footnotetext{
${ }^{1}$ Reduced vertical gait dynamic is supposed to be one key feature of depressed gait, as found in previous research (Michalk et al. 2009). This is in line with the finding that depressed patients tend to walk with a lifting motion of their legs, whereas normal control subjects propel themselves forward with a more pronounced vertical gait (Sloman et al. 1982). Thus, the enhanced vertical dynamic feature seen in non-depressed individuals stems from a generally more dynamic gait pattern.
} 
Table 1 Sample Characteristics

\begin{tabular}{lccr}
\hline & Healthy controls $(\mathrm{n}=36)$ & MDD patients $(\mathrm{n}=35)$ & $\mathrm{p}$-Value \\
\hline Age, M (SD) & $39.06(10.00)$ & $39.57(12.30)$ & $\mathrm{p}=0.847^{1}$ \\
Male sex, N (\%) & $15(42)$ & $13(37)$ & $\mathrm{p}=0.697^{2}$ \\
BMI, M (SD) & $24.48(3.56)$ & $24.72(4.71)$ & $\mathrm{p}=0.805^{2}$ \\
BDI, M (SD) & $2.50(3.26)$ & $28.69(10.55)$ & $\mathrm{p}<0.001^{2}$ \\
\hline
\end{tabular}

Note: $1=\chi^{2}$ Test, $2=\mathrm{t}$-Test

\section{Method}

\section{Participants}

51 patients and 50 non-depressed controls (matched for sex, age, and body mass index) participated in this study. Patients were recruited from outpatient clinics and local psychotherapists in Berlin, Germany. Controls were recruited from advertisings in local newspapers. The data of 14 control participants and 16 patients were lost due to hardware failure either in the palm handhelds, the accelerometer, or in the time-based assignment of accelerometer and subjective mood data. Thus, the final sample consisted of 36 controls ( $\mathrm{n}=15$ male, $\mathrm{n}=21$ female), and 35 depressed patients ( $\mathrm{n}=13$ male, $\mathrm{n}=22$ female), with age ranging from 18-60 $(\mathrm{M}=39.31, \mathrm{SD}=11.12)$ years, and body mass index $(\mathrm{kg} /$ $\mathrm{m}^{2}$ ) ranging from $18-39(\mathrm{M}=24.60, \mathrm{SD}=4.14)$. Controls and patients did not differ with respect to sex, age, and body mass index (see Table 1). Patients and controls were diagnosed with a standardized diagnostic interview for DSM-IV disorders (Schneider and Margraf 2011) by trained and certified psychotherapists. All patients fulfilled DSM-IV criteria for current diagnosis of major depression $(n=25$ recurrent major depression, $\mathrm{n}=6$ single episode of major depression). In total, $n=22$ patients had additional diagnoses $(n=10$ one additional diagnosis, $n=9$ two, $n=2$ three, $n=1$ five additional diagnoses). Among these comorbid diagnoses, anxiety disorders were the most frequent $(n=13)$. Non-depressed controls had no current and no history of mental disorders. Patients had significantly higher scores on the Beck Depression Inventory (BDI) than controls (see Table 1). A total of $\mathrm{n}=18$ patients took psychotropic medication (SSNRI $\mathrm{n}=3$, SSRI $n=7$, tricyclic antidepressant $n=3$, antiepileptic medication $n=4$, neuroleptics $n=4$, dopaminergic antagonists $n=1$, MAO inhibitors $n=2$, amphetamines $n=3$ ). Most patients received a single substance $(n=10)$, seven patients two types of medication simultaneously, and one patient four types of medication.

\section{Measures}

\section{Assessment of Gait Parameters and Body Motility}

Body activity was assessed with two accelerometers, in accordance with published recommendations (Foerster and Fahrenberg 2000). One accelerometer (three-channel: sagittal, longitudinal, transversal axis) was placed over the participant's trunk and another accelerometer (one-channel: sagittal axis) placed on the outer part of the upper right leg (Foerster and Fahrenberg 2000; Reichert et al. 2015) (both accelerometers provided by Vitamove, NL). Acceleration data (range: $\pm 6 \mathrm{~g}$ ) were sampled continuously with a sampling rate of $128 \mathrm{~Hz}$. From the raw data, periods of active (i.e., activities in upright position, for example walking, running, bicycling) and sedentary behavior (i.e., lying, sitting) were identified automatically offline according to published movement pattern identification algorithms (Bussmann et al. 2001; overview in Bussmann et al. 2009) using commercial software (Vitascore, Temec, NL). The participant's nonspecific amount of overall movement intensity (i.e., body motility) was calculated in accordance with previous reports (von Haaren et al. 2016) using customized scripts (Matlab). For periods of walking, four specific gait parameters were additionally extracted, corresponding to the laboratory study of Michalak et al. (2009).

In brief, body posture was calculated as the arctangent of the low pass filtered $(0.5 \mathrm{~Hz})$ raw signal from the sagittal axis of the trunk sensor (range $\pm 180^{\circ}$ ). Positive values of body posture signify forward bowing of the participants. Lateral swaying movements and vertical up-and down movements were estimated from the transversal and longitudinal axis of the trunk sensor, respectively, and calculated using customized scripts.

Both signals were bandpass-filtered within the frequency range of walking (i.e., $0.5-3 \mathrm{~Hz}$ ), rectified and smoothed using a moving average function (width $1 \mathrm{~s}$ ). Walking speed was calculated with commercial software (for details see Vitascore, Temec, NL).

\section{Self-Report Measures}

Assessment of current mood. Self-report measures were assessed with Palm Tungsten T3 handhelds running the 
Fig. 1 Illustration of the sampling of data. Red boxes represent segments during which a participant was walking. These segments were aggregated across the 55 min prior to each affect self-report to yield the respective mean gait parameters

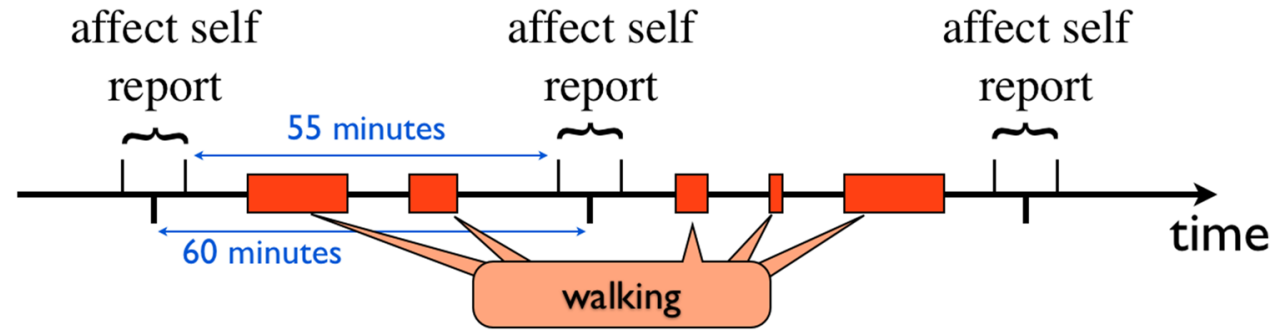

freely available Experience Sampling Program software (ESP, version 4.0). Handhelds were configured to request the participants' input regularly in $1-\mathrm{h}$ intervals by releasing a series of single beeps (i.e., time-based assessment, see Shiffman et al. 2008). Upon participants' response, a number of questions assessing the participants' mood and physical activity were released. Questions and assessment of positive and negative affect was assessed based on the procedure described earlier (Mata et al. 2011). In brief, participants were asked to indicate on a 4-point scale from "not at all" to "completely" how anxious, sad, disgusted, angry, guilty, ashamed, happy, frustrated, excited, alert and active they were at that moment. ${ }^{1}$

Beck Depression Inventory (BDI). We used the BDI (German version by Hautzinger et al. 2006) to assess depressive symptoms by self-report. The BDI is a widely used 21-item measure covering affective, cognitive, motivational, behavioral, and biological symptoms of depression with good psychometric properties (Beck et al. 1988).

\section{Procedure}

To increase reliability, data were assessed on two consecutive days, preferably a weekday and a weekend day (see Buchowski et al. 2004). Distribution of weekdays and weekend days did not differ between patients and non-depressed controls, $\mathrm{p}=0.318$. On the day prior to the first day of data assessment, participants were introduced to set up the two accelerometers and to the use of the Palm handhelds, and were asked to fill in the BDI questionnaire. Participants were informed that the aim of the study was to assess the association between physiological parameters and mood. They were instructed to wear the accelerometers during the entire day until going to sleep and to put on the accelerometer again in the morning of the next day as early as possible. They were then told that they should respond to every prompt of the Palm handheld, whenever possible, but that they should refrain from responding while driving a car. Participants were informed that the Palm would prompt them every hour from 8:00 am to 10:00 pm on both days (Total response to prompts did not differ between non-depressed controls, $89,4 \%$ and MDD patients, 87,9\%, $\mathrm{p}=0.666)$. At the end of the second day, participants returned the equipment and were thanked for participating.

\section{Data Reduction and Statistical Analysis}

First, those segments were extracted during which the participant was walking (as identified by movement pattern identification algorithm). Data, where the participants were running were excluded from analyses (Bussmann et al. 2001). Figure 1 exemplifies the chronology of movement detection within the individual data sets of each participant. These segments were subsequently used for further analyses. To evaluate group differences between patient and control participants, we computed the mean of each gait parameter per participant during these walking phases over the entire two days of data assessment (i.e., mean overall movement intensity, body posture, lateral swaying movements, vertical up-and-down movements and walking speed). Simple t-tests were performed to evaluate differences between patients and non-depressed controls. In addition, mean positive and negative affect was calculated by averaging all responses over the entire 2 days of data assessment. Simple t-tests were calculated to assess differences between patients and controls in mean overall positive and negative affect.

To evaluate the predictive value of the four gait patterns on self-reported mood, again those segments were used during which the participant was walking. Because affect selfreport was requested every $60 \mathrm{~min}$, and five minutes time had to be allotted to the respective subsequent self-reporting, data were analyzed for the remaining 55 min of each 60 -min interval. For each of the identified walking segments within the 55 min prior to a respective self-report, data of each of the four gait parameters (i.e., 'body posture', 'vertical movement', 'lateral movement', and 'walking speed') were averaged. Additionally, overall movement intensity was calculated by aggregating overall movement intensity data over the entire 55 min of each interval.

The study duration of two days provided a number of self-reports and related gait parameters for each participant. This hierarchical dataset with repeated measures was modeled using multilevel regression, with self-reported positive and negative affect as the two dependent variables, the four gait parameters as the fixed effects, and participant as the 
random effect. The intercepts as well as the slopes of the fixed effects were allowed to vary freely, and therefore 'participant intercept' and 'participant slope' were entered as the random effects into the models.

We computed a total of 10 multilevel models considering the four gait parameters (body posture, vertical up-anddown movements, lateral swaying movements, and walking speed) predicting both affect variables (positive and negative affect). Gait parameters as observed during the $55 \mathrm{~min}$ preceding the respective self-report assessments were used as predictors. Within each model, affect-ratings from the preceding self-report assessment (i.e., positive or negative affect one hour ago) were entered as a covariate. We additionally added group membership (i.e., patients vs. controls) and the interaction of group membership with the respective gait parameter to test whether the gait parameters differentially predicted mood within depressed patients or nondepressed controls. Finally, overall movement intensity was used as a covariate in each model to test whether the predictive power of gait parameters would exceed the mere overall motor activity level. Taken together, this approach enables, under control of participants' overall movement intensity, if a change in mood from one hour to another is significantly predicted by a previous change in the four gait parameters. Since previous research has shown that MDD patients and non-depressed controls most likely differ in overall positive and negative affect (e.g., Mata et al. 2011), adding group membership also enabled us to control for these overall differences in mood in our multilevel analyses. Thus, the models in our study aimed at analyzing the association between measures of mood (i.e., positive and negative affect) and gait patterns as recorded over time.

Finally, a set of control analyses was performed to account for the possibility of a bi-directional association of affect and gait. To this end, we computed a total of 10 multilevel models considering positive and negative affect predicting the four gait parameters (body posture, vertical up-and-down movements, lateral swaying movements, and walking speed) and overall movement intensity as observed during the $55 \mathrm{~min}$ after the respective self-report assessments. Within each model, affect ratings of the preceding self-report assessment (i.e., positive or negative affect one hour ago) were entered as a covariate. Again, we included group membership (i.e., MDD patient vs. controls) and its interactions with the respective predictors (in these models, negative and positive affect) as factors to our analyses. All statistical analyses were performed using JMP Pro 14 statistical software (SAS Institute Inc.).
Table 2 Mean (M) and standard deviations (SD) of gait parameters, negative and positive affect of healthy controls and MDD patients

\begin{tabular}{llrlll}
\hline & \multicolumn{2}{l}{ MDD patients } & & \multicolumn{2}{c}{ Healthy controls } \\
\cline { 2 - 3 } & M & SD & & M & SD \\
\hline Body posture & 8.47 & 10.39 & & 2.93 & 8.12 \\
Vertical movements & 0.30 & 0.06 & & 0.35 & 0.07 \\
Lateral sway & 0.07 & 0.01 & & 0.07 & 0.01 \\
Walking speed & 1.85 & 0.51 & & 2.21 & 0.53 \\
Positive affect & 0.84 & 0.45 & & 1.49 & 0.34 \\
Negative affect & 0.53 & 0.53 & & 0.11 & 0.21 \\
\hline
\end{tabular}

\section{Results}

\section{Differences in Overall Mood Between Patients and Controls}

MDD patients reported less overall positive affect (i.e., mean PA over the entire two days of data assessment $), t(69)=6.89$, $\mathrm{p}<0.001, \mathrm{~d}=1.63$, and more negative affect (i.e., mean NA over the entire two days of data assessment), $\mathrm{t}(69)=4.43$, $\mathrm{p}<0.001, \mathrm{~d}=1.04$, as compared to non-depressed controls over the two days of data acquisition.

\section{Differences in Gait Parameters Between Patients and Controls}

Table 2 shows mean gait parameters averaged for the 2-day assessment period. Results indicate that over the entire assessment period (i.e., day one and day two), depressed patients showed significantly more forward-leaning slumped body posture, $\mathrm{t}(69)=-2.51, \mathrm{p}<0.05, \mathrm{~d}=0.59$, $95 \%$ CI $[1.13,9.95]$, significantly less vertical up-anddown movements, $\mathrm{t}(69)=3.07, \mathrm{p}<0.01, \mathrm{~d}=0.73,95 \% \mathrm{CI}$ $[-0.07,-0.02]$, and significantly lower walking speed, $\mathrm{t}(69)=-2.91, \mathrm{p}<0.01, \mathrm{~d}=0.69,95 \%$ CI $[-0.61,-0.11]$. Depressed patients and controls did not differ in lateral swaying movements, $\mathrm{t}(69)=-0.10$, n.s. (see Fig. 2).

To control for the possible influence of psychotropic medication, we compared gait parameters between medicated and unmedicated MDD patients. Results indicate that psychotropic medication did not influence body, posture, lateral swaying movements, and walking speed (all p >0.19), while patients taking psychotropic medication exhibited significantly less vertical up-and-down movements than patients not taking medication, $\mathrm{t}(33)=2.61, \mathrm{p}=0.014$.

To test whether psychotropic medication could explain the significant difference in vertical up-and-down movements between non-depressed controls and MDD patients, ANOVAs controlling for psychotropic medication were run. Results indicate that less vertical up-and-down movements in MDD patients in comparison to non-depressed 

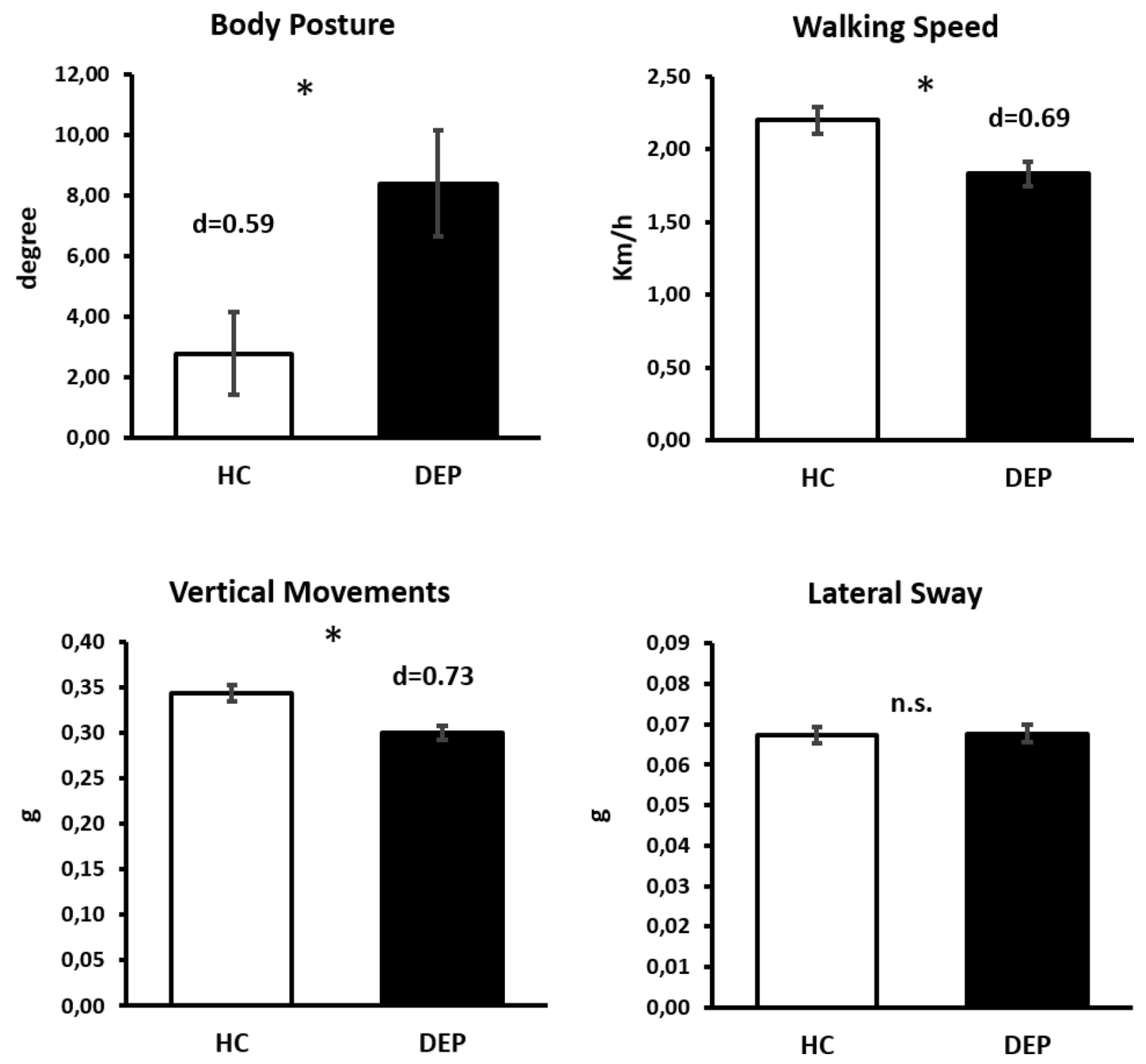

Fig. 2 Mean ( \pm SEM) body posture (upper left), walking speed (upper right), vertical up- and down movements (lower left) and lateral swaying movements (lower right) for healthy controls (HC) and MDD patients (DEP). (* p <0.05)

controls was not explained by psychotropic medication use, $\mathrm{F}(1,67)=5.58, \mathrm{p}=0.021$.

\section{Prediction of Affect by Gait Parameters}

The models for positive affect are given in Table 3. The total number of observations in each model was $n=1435$. Table 1 shows that vertical up-and-down movements and walking speed were predictive of positive affect. When overall movement intensity was additionally entered as a control variable, vertical motility and walking speed remained significant predictors of positive affect (Models 5 and 9). We did not find significant interactions between group membership (i.e., patients vs. controls) and any of the four gait parameters, indicating that the association between gait und subsequent mood could be observed in non-depressed as well as in depressed individuals. Finally, higher overall movement intensity (in the absence of any other predictor) was also significantly associated with higher positive affect (Model 2). We also tested the association of the respective gait parameters on subsequent changes in negative affect (Table 4). None of the models showed any significant association between gait and subsequent negative affectivity (all $\mathrm{p}>0.10)^{2}$

\footnotetext{
2 All hierarchical models have also been calculated using personmean centered effects. We obtained comparable results: walking speed and vertical up-and-down movements predicted positive mood, all other parameters showed no significant associations.
} 


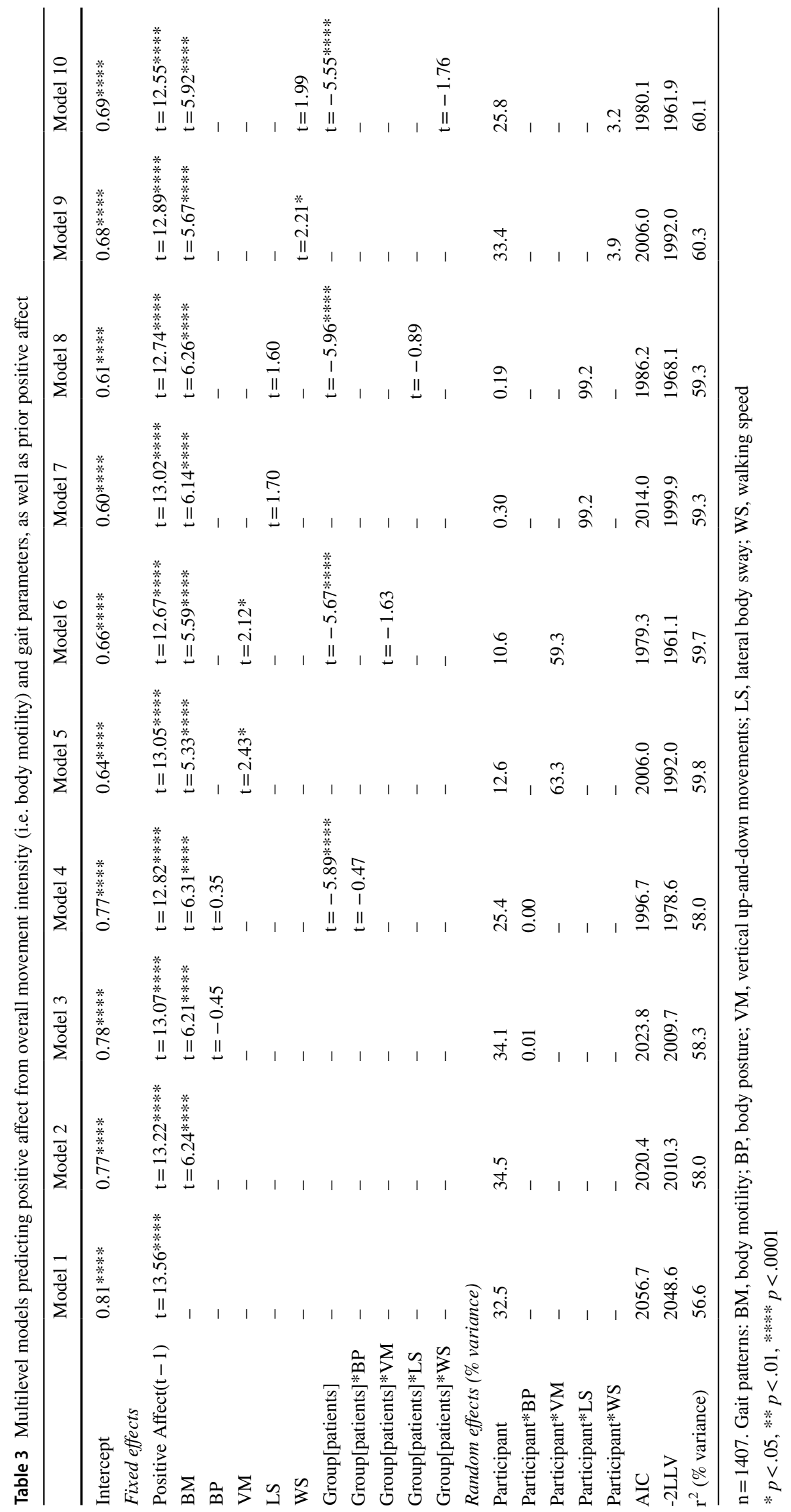




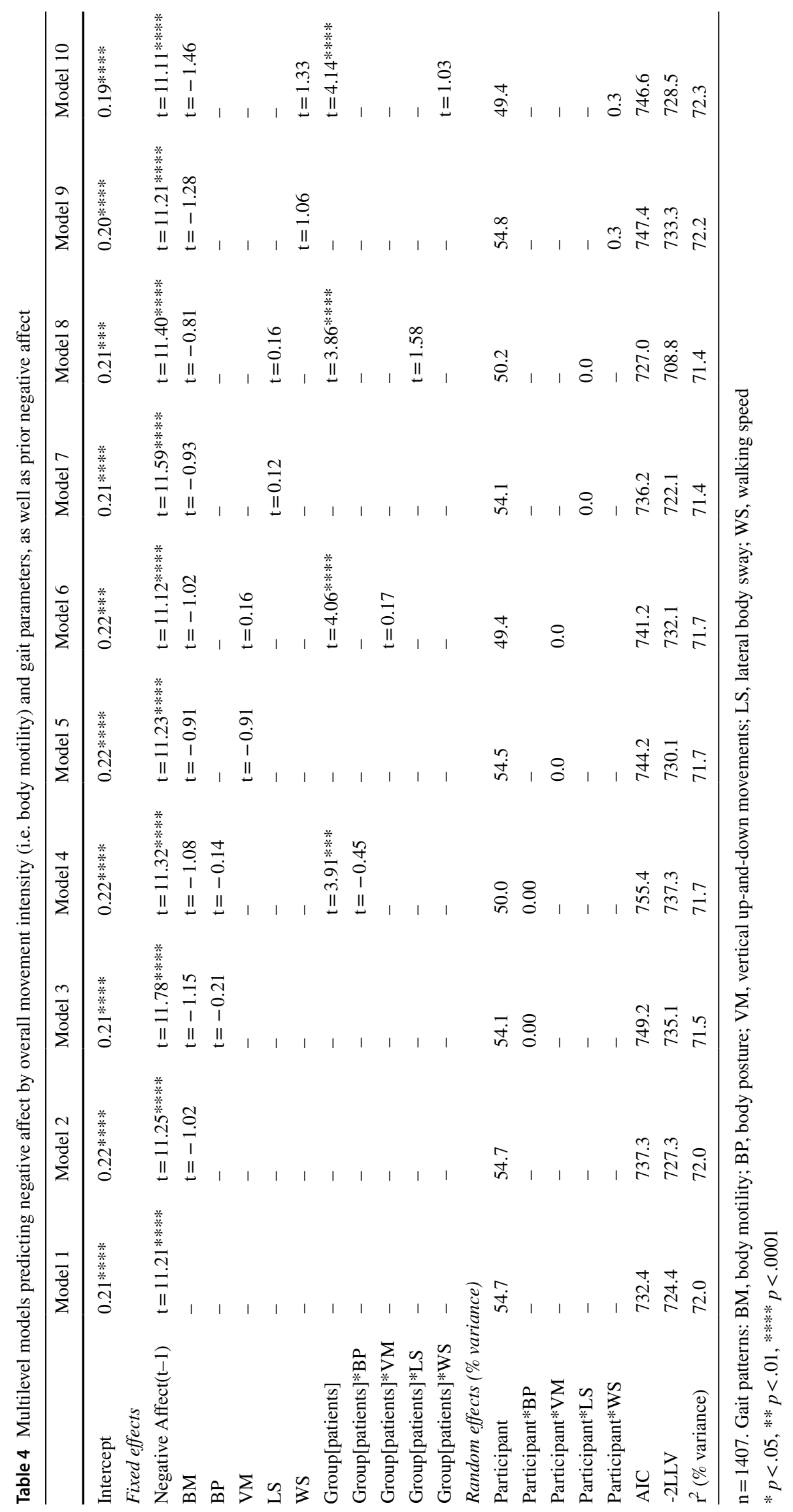




\section{Prediction of Gait Parameters by Affect}

Finally, we evaluated the association of mood with subsequent changes in gait parameters. None of the models showed any significant association between positive affect or negative affect and subsequent changes in gait or overall movement intensity (all $\mathrm{p}>0.10$ ).

\section{Discussion}

The present research had two major goals. First, we aimed at replicating, in everyday life, findings on gait characteristics of depressed individuals previously found in the laboratory. In their study Michalak et al. (2009) demonstrated that in the laboratory patients with MDD as compared to nondepressed controls showed reduced walking speed, reduced arm-swing, smaller amplitudes of vertical movements of the upper body, larger lateral body sway, and a more slumped and forward-leaning posture. Using two accelerometers in the current study, we were able to measure four of these five gait parameters in everyday life (arm swing was not measured). Replicating the results of Michalak et al. (2009), our results confirmed that depressed individuals indeed walked more slowly, with a more slumped posture and with smaller amplitudes of vertical up-and-down movements than control participants. Thus, differences in posture between nondepressed controls and patients with MDD found in the laboratory were also present under more naturalistic, everyday life conditions. Correspondingly, the current results support the idea that depression is embodied in the way people walk (Michalak et al. 2009). Importantly, the differences found in terms of gait, cannot be explained by an overall lower level of energetic drive, a major symptom of depression and thus a possible confounder to the current data. We found that overall levels of movement intensity did not differ between patients suffering from MDD and non-depressed controls. Thus, simple differences in energetic drive, as represented by changes in overall body motility, cannot account for the differences in gait we found in our data (Table 5).

Contrary to walking speed, vertical up-and-down movements and body posture, we could not replicate previous laboratory findings on larger amplitudes of lateral body swaying in MDD. This might indicate that depressed individuals do not show larger lateral body sway when walking in everyday life. However, this conclusion should be drawn with caution. In the laboratory, the participants walked a straight line (Michalak et al. 2009). In contrast, in natural environments people tend to often slightly switch their direction when walking. This may produce noise especially when measuring lateral movements of the upper body. In the present study, this may have masked the differences in lateral body sway between depressed and control participants. In sum, our findings indicate that both in the laboratory and under naturalistic conditions depressed individuals showed a specific walking style. To date almost all research on embodiment is conducted in laboratory settings (Elkjær et al. 2020). Thus, the current findings extend this literature and show that depression is accompanied by altered gait patterns also in naturalistic settings.

The second aim of our study was to further our understanding of the longitudinal dynamics of body-mood interaction and their putative disturbances in depression. Therefore, we investigated whether the gait parameters averaged over a 55-min period could predict subsequent mood and vice versa. We found that walking speed and vertical up-anddown movement significantly predicted changes in future positive mood. In detail, faster walking and larger vertical amplitudes during walking were associated with subsequent reports of increased positive mood. Controlling for overall movement intensity in our analyses did not change this association, thus rendering unlikely the possibility that the increase in positive mood was merely attributable to an increase in general, unspecific activity levels. No effects were found for the association between mood (positive and negative) and subsequent gait patterns.

Previous research has demonstrated an association between overall movement intensity and enhanced positive mood (meta-analyses in Chan et al. 2019). To the best of our knowledge, our study is the first to show that beyond unspecific general activity level, also gait parameters significantly add to this relationship and impact mood in everyday life.

Overall, and in line with previous reports (Mata et al. 2011), we found that MDD patients reported significantly more negative and significantly less positive affect over the course of the 2-day data assessment. In the contrary, we did not find significant differences in the association of mood and gait parameters between depressed individuals and control participants, indicating that the association between walking speed as well as vertical movements of the body with positive mood may constitute a general pattern that is independent of the diagnostic status of a person. However, it should be noted that the sample size of our study may have been insufficient to reliably detect subtle group differences between depressed and non-depressed individuals with respect to the prediction of mood by gait parameters. Specifically, previous research (Mata et al. 2011) has found a significantly stronger association between mood change and body activity in depressed individuals. Therefore, future research should utilize larger samples to investigate potential group differences in the temporal dynamics between gait parameters and mood.

In contrast to walking speed and vertical movements, posture did not predict positive mood. This result stands in contrast to our cross-sectional analysis which revealed significant differences in posture between depressed and control 


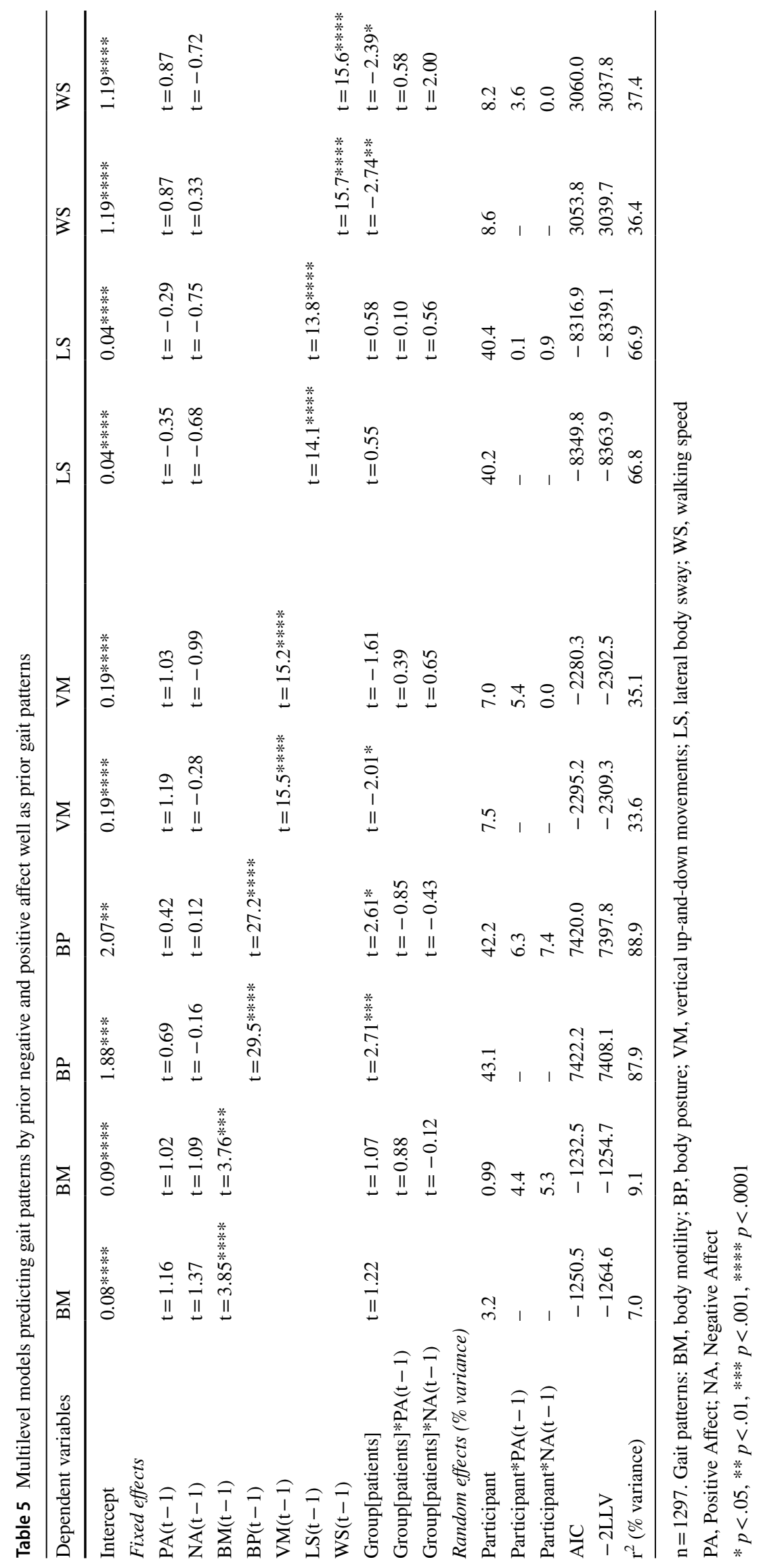


participants. Possibly, some gait parameters (speed, vertical movements) may influence positive mood in the time frame we used for our analysis (one hour), whereas effects of posture may follow another time frame. In this case, a more extended period would be needed to observe effects of posture on mood. Since experimental studies have shown effects of posture on affective variables (Elkjær et al. 2020; Michalak et al. 2014), future studies should investigate whether the present null findings may be attributed to the specific methodological framework used in the present study.

A particularly puzzling result of our study was that negative mood was not predicted by any of the four gait parameters. This result was not consistent with evidence reported by a previous meta-analysis (Elkjær et al. 2020). This meta-analysis showed that experimentally manipulating bodily displays in the laboratory has broad effects on mood, including positive as well as negative emotional states. However, our results are in accordance with another study (Mata et al. 2011) which reported that self-initiated physical activity in everyday life influenced positive but not negative affect in depressed patients and neverdepressed individuals. These contrasting findings indicate that more research in naturalistic settings is needed to investigate which kind of bodily displays have effects on positive and negative mood in depressed individuals. The fact that we mainly found associations of gait with positive affect might also encourage future research to investigate the role of gait in everyday life of patients with bipolar disorders. Future research in this direction is also suggested by laboratory findings showing gait alterations (e.g., increase walking speed, enhanced force during gait) during hypomanic phases (Kang et al. 2018).

To sum up, our results support the expectation that embodiment in depression is also observable in naturalistic settings. The state of depression is bodily manifested in the way people walk. Moreover, at least some of the features of gait are longitudinally associated with (positive) mood. The latter finding might indicate that gait patterns have a causal effect on mood, as is postulated by theories on bodymind feedback loops in the etiology of depression (Teasdale 1999). However, a limitation of our study is that no firm causal interpretation of our results is possible (i.e., we did not experimentally manipulate gait). Thus, we cannot rule out that a third variable is responsible for the observed temporal associations between gait and subsequent positive affect. Despite this limitation, experimental research showing that manipulating motor displays affect a broad range of affective variables (Elkjær et al. 2020) and more specifically the results of Michalak et al. (2015) showing that changing people's walking style affects mood are nonetheless in accord with a causal interpretation of the temporal associations between gait and subsequent positive affect.
Our analyses on the predictive value of positive (and negative) affect on subsequent gait patterns did not reveal any significant effects. This finding argues against a bidirectional association of positive affect and gait but rather indicates a unidirectional temporal association between gait parameters predicting changes in positive affect. However, future research should verify the robustness of this null finding using other methodological approaches (e.g., experimental studies, studies using other time frames to investigate possible effects of mood on gait).

Another limitation of our study was that due to restrictions of the current statistical approach (i.e., collinearity of predictors, limited sample size) it was not possible to include all parameters in one model to test for the unique contribution of each gait parameter on the association with current mood. To this end, future approaches should gain larger samples to identify the principal components-or the leading force-responsible for the association of gait and changes in mood.

Bearing these limitations in mind, the present results might open up new perspectives for the treatment of MDD. While existing activity-based treatments of depression (e.g., Rosenbaum et al. 2014) or those targeting behavior activation (e.g., Lejuez et al. 2011) primarily focus on increasing level of activity, our findings indicate that not (only) the level of activity itself is crucial (we found no effects of overall movement intensity in our study), instead the way individuals walk might be an important factor for the dynamics of their mood. Therefore, interventions specifically targeting walking style modifications, especially assisting patients with MDD to walk more vigilantly and with intensified vertical dynamics, might be a promising treatment option warranting future research. Interventions that focus on the bodily level may be especially promising for patients who do not profit from existing verbally-based interventions. Thus, future research should investigate the effects of a systematic and extended training of non-depressed gait patterns for patients with MDD. These studies should measure a broad range of outcomes including mood as well as symptom reduction and quality of life. We foresee that using an embodiment perspective in the treatment of MDD will have the potential to widen the scope of treatment options for this debilitating condition.

Acknowledgements This study was funded by German Research Foundation (DFG) grant Mi 700/5-1 awarded to JM and German Research Foundation grant AD 452/1 awarded to DA. We would like to thank Babette Renneberg for making it possible to recruit patients in the outpatient clinic of the Free University Berlin.

Author contributions DA and JM contributed to the study conception and design. Material preparation, and data collection were done by $\mathrm{HN}$, data analysis and reporting was performed by WT. The first draft of the manuscript was written by DA and JM and all authors commented 
on previous versions of the manuscript. All authors read and approved the final manuscript.

Funding Open Access funding enabled and organized by Projekt DEAL..

\section{Compliance with Ethical Standards}

Conflict of Interest The authors declare that they have no conflict of interest.

Informed Consent All participants gave written informed consent to the procedures. The study was approved by the local Ethics Committee and was conducted in agreement with the Declaration of Helsinki.

Animal rights No animals were tested within the current study.

Open Access This article is licensed under a Creative Commons Attribution 4.0 International License, which permits use, sharing, adaptation, distribution and reproduction in any medium or format, as long as you give appropriate credit to the original author(s) and the source, provide a link to the Creative Commons licence, and indicate if changes were made. The images or other third party material in this article are included in the article's Creative Commons licence, unless indicated otherwise in a credit line to the material. If material is not included in the article's Creative Commons licence and your intended use is not permitted by statutory regulation or exceeds the permitted use, you will need to obtain permission directly from the copyright holder. To view a copy of this licence, visit http://creativecommons.org/licenses/by/4.0/.

\section{References}

Beck, A. T., Steer, R. A., \& Carbin, M. G. (1988). Psychometric properties of the Beck Depression Inventory: Twenty-five years of evaluation. Clinical Psychology Review, 8(1), 77-100. https://doi. org/10.1016/0272-7358(88)90050-5.

Buchowski, M. S., Acra, A., Majchrzak, K. M., Sun, M., \& Chen, K. Y. (2004). Patterns of physical activity in free-living adults in the southern united states. European Journal of Clinical nutrition, $58,828-837$.

Bussmann, J. B. J., Ebner-Priemer, U. W., \& Fahrenberg, J. (2009). Ambulatory activity monitoring. European Psychologist, 14(2), 142-152. https://doi.org/10.1027/1016-9040.14.2.142.

Bussmann, J. B. J., Martens, W. L. J., Tulen, J. H. M., Schasfoort, F. C., van den Berg-Emons, H. J. G., \& Stam, H. J. (2001). Measuring daily behavior using ambulatory accelerometry: The activity monitor. Behavior Research Methods, Instruments, \& Computers, 33(3), 349-356. https://doi.org/10.3758/bf03195388.

Chan, J. S. Y., Liu, G., Liang, D., Deng, K., Wu, J., \& Yan, J. H. (2019). Special issue-Therapeutic benefits of physical activity for mood: A systematic review on the effects of exercise intensity, duration, and modality. The Journal of Psychology, 153(1), 102-125. https ://doi.org/10.1080/00223980.2018.1470487.

Cuddy, A. J. C., Schultz, S. J., \& Fosse, N. E. (2018). P-curving a more comprehensive body of research on postural feedback reveals clear evidential value for power-posing effects: Reply to Simmons and Simonsohn (2017). Psychological Science, 29(4), 656-666. https ://doi.org/10.1177/0956797617746749.

Elkjær, E., Mikkelsen, M. B., Michalak, J., Mennin, D. S., \& O’Toole, M. S. (2020). Expansive and contractive postures and movement: A systematic review and meta-analysis of the effect of motor displays on affective and behavioral responses. Perspectives on Psychological Science. https://doi.org/10.1177/174569162091935 8.

Foerster, F., \& Fahrenberg, J. (2000). Motion pattern and posture: Correctly assessed by calibrated accelerometers. Behavior Research Methods, Instruments, \& Computers, 32(3), 450-457. https://doi. org/10.3758/bf03200815.

Hautzinger, M., Keller, F., \& Kühner, C. (2006). Beck depressionsinventar (BDI-II): Harcourt Test Services.

Kang, G. E., Mickey, B. J., McInnis, M. G., Krembs, B. S., \& Gross, M. M. (2018). Motor behavior characteristics in various phases of bipolar disorder revealed through biomechanical analysis: Quantitative measures of activity and energy variables during gait and sit-to-walk. Psychiatry Research, 269, 93-101. https:// doi.org/10.1016/j.psychres.2018.08.062.

Lejuez, C. W., Hopko, D. R., Acierno, R., Daughters, S. B., \& Pagoto, S. L. (2011). Ten year revision of the brief behavioral activation treatment for depression: Revised treatment manual. Behavior Modification, 35(2), 111-161. https://doi.org/10.1177/01454 45510390929.

Lemke, M. R., Wendorff, T., Mieth, B., Buhl, K., \& Linnemann, M. (2000). Spatiotemporal gait patterns during over ground locomotion in major depression compared with healthy controls. Journal of Psychiatric Research, 34(4), 277-283. https://doi.org/10.1016/ S0022-3956(00)00017-0.

Lyons, N., Graser, J., Dietrich, D., Müller, B. \& Michalak, J. (2021). Reduced jumping to conclusion bias after experimentally induced enhancement of subjective body-boundaries in psychosis. Psychopathology. https://doi.org/10.1159/000513271.

Mata, J., Thompson, R. J., Jaeggi, S. M., Buschkuehl, M., Jonides, J., \& Gotlib, I. H. (2011). Walk on the bright side: Physical activity and affect in major depressive disorder. Journal of Abnormal Psychology, 121(2), 297-308. https://doi.org/10.1037/a0023533.

Mathews, A., \& MacLeod, C. (2005). Cognitive vulnerability to emotional disorders. Annual Review of Clinical Psychology, 1(1), 167-195. https://doi.org/10.1146/annurev.clinpsy.1.102803.14391 6.

Matt, G. E., Vázquez, C., \& Campbell, W. K. (1992). Mood-congruent recall of affectively toned stimuli: A meta-analytic review. Clinical Psychology Review, 12(2), 227-255. https://doi. org/10.1016/0272-7358(92)90116-P.

Michalak, J., Chatinyan, A., Chourib, H., \& Teismann, T. (2018). The impact of upward versus downward movement patterns on memory characteristics of depressed individuals. Psychopathology, 51(5), 326-334. https://doi.org/10.1159/000492788.

Michalak, J., Mischnat, J., \& Teismann, T. (2014). Sitting posture makes a difference-Embodiment effects on depressive memory bias. Clinical Psychology \& Psychotherapy, 21(6), 519-524. https ://doi.org/10.1002/cpp.1890.

Michalak, J., Rohde, K., \& Troje, N. F. (2015). How we walk affects what we remember: Gait modifications through biofeedback change negative affective memory bias. Journal of Behavior Therapy and Experimental Psychiatry, 46, 121-125. https://doi. org/10.1016/j.jbtep.2014.09.004.

Michalak, J., Troje, N. F., Fischer, J., Vollmar, P., Heidenreich, T., \& Schulte, D. (2009). Embodiment of sadness and depression-Gait patterns associated with dysphoric mood. Psychosomatic Medicine, 71(5), 580-587. https://doi.org/10.1097/PSY.0b013e3181 a2515c.

Paleacu, D., Shutzman, A., Giladi, N., Herman, T., Simon, E. S., \& Hausdorff, J. M. (2007). Effects of pharmacological therapy on gait and cognitive function in depressed patients. Clinical Neuropharmacology, 30(2), 63-71. https://doi.org/10.1097/01.wnf.00002 40949.41691.95.

Reichert, M., Lutz, A., Deuschle, M., Gilles, M., Hill, H., Limberger, M. F., \& Ebner-Priemer, U. W. (2015). Improving motor activity 
assessment in depression: Which sensor placement, analytic strategy and diurnal time frame are most powerful in distinguishing patients from controls and monitoring treatment effects. PLoS ONE, 10(4), e0124231. https://doi.org/10.1371/journ al.pone. 0124231 .

Rosenbaum, S., Tiedemann, A., Sherrington, C., Curtis, J., \& Ward, P. (2014). Physical activity interventions for people with mental illnesses: A systematic review and meta-analysis. The Journal of clinical Psychiatry, 75, 964-974.

Schneider, S., \& Margraf, J. (2011). Diagnostisches Interview bei psychischen Störungen (DIPS für DSM-IV-TR) (Vol. 4th, completely (revised). Berlin: Springer.

Shiffman, P., Stone, A. A., \& Hufford, M. R. (2008). Ecological momentary assessment. Annual Review of Clinical Psychology, 4(1), 32. https://doi.org/10.1146/annurev.clinpsy.3.022806.09141 5.

Simmons, J. P., \& Simonsohn, U. (2017). Power posing: P-curving the evidence. Psychological Science, 28(5), 687-693. https://doi. org/10.1177/0956797616658563.

Sloman, L., Berridge, M., Homatidis, S., Hunter, D., \& Duck, T. (1982). Gait patterns of depressed patients and normal subjects. The American Journal of Psychiatry, 139(1), 94-97. https://doi. org/10.1176/ajp.139.1.94.

Sloman, L., Pierrynowski, M., Berridge, M., Tupling, S., \& Flowers, J. (1987). Mood, depressive illness and gait patterns. The Canadian Journal of Psychiatry, 32(3), 190-193. https://doi. org/10.1177/070674378703200306.

Teasdale, J. D. (1999). Multi-level theories of cognition-emotion relations. In T. Dalgleish \& M. Power (Eds.), Handbook of cognition and emotion (pp. 665-681). Chichester: Wiley.
Teasdale, J. D., \& Barnard, P. J. (1993). Affect, cognition and change: Remodelling depressive thought. Hove: Lawrence Erlbaum Associates.

Tschacher, W., Giersch, A., \& Friston, K. (2017). Embodiment and schizophrenia: A review of implications and applications. Schizophrenia Bulletin, 43(4), 745-753. https://doi.org/10.1093/schbu $1 /$ sbw220.

von Haaren, B., Ottenbacher, J., Muenz, J., Neumann, R., Boes, K., \& Ebner-Priemer, U. (2016). Does a 20-week aerobic exercise training programme increase our capabilities to buffer real-life stressors? A randomized, controlled trial using ambulatory assessment. European Journal of Applied Physiology, 116(2), 383-394. https://doi.org/10.1007/s00421-015-3284-8.

Wagenmakers, E.-J., Beek, T., Dijkhoff, L., Gronau, Q. F., Acosta, A., Adams, R. B., et al. (2016). Registered replication report: Strack, Martin, \& Stepper (1988). Perspectives on Psychological Science, 11(6), 917-928. https://doi.org/10.1177/1745691616674458.

Winkielman, P., Niedenthal, P., Wielgosz, J., Eelen, J., \& Kavanagh, L. C. (2015). Embodiment of cognition and emotion APA handbook of personality and social psychology, Volume 1: Attitudes and social cognition (pp. 151-175). Washington, DC: American Psychological Association.

Publisher's Note Springer Nature remains neutral with regard to jurisdictional claims in published maps and institutional affiliations. 